\title{
Comments on: Process modeling for slope and aspect with application to elevation data maps
}

\author{
Giovanna Jona Lasinio ${ }^{1}$ (D) - Gianluca Mastrantonio ${ }^{2}$ (D) \\ Published online: 12 November 2018 \\ c) Sociedad de Estadística e Investigación Operativa 2018
}

First of all, we wish to congratulate the authors for the very interesting contribution. Since in the literature no stochastic models that properly describe the computation of aspect and slope were available, the proposal of the authors is really helpful. Models that are based on the real physical meaning of variables are usually much more useful than others where the interpretation of model's elements may be quiet awkward.

The authors do not develop at this stage a dynamic version of their proposal. In their setting indeed it does not seem reasonable to add a dynamical part. On the other hand, adding a temporal dynamic may, in specific applications at small scale, help develop models for data describing the development of unstable geological formations such as landslide (inclinometer and extensometer sensors data for example). Given the use of a GP in the modeling of elevation and $\Delta \mu$, this step seems straightforward. The spatial GP can be replaced by a space-time GP and then the same developments should apply.

Very interesting will be to see how the authors will handle large datasets. We would like to read some comments on, for example, the use of nearest neighbor Gaussian processes (NNGP) or some other law rank methods in this problem. We imagine that using NNGP on the elevation data and hence on $\Delta \mu$ will not change anything in the theoretical development proposed. The main issue that we can see is related to how the approximation on the elevation surface affects the estimates of slope and aspect.

We have a few questions:

- do the authors have any idea on how the uncertainty on elevation may affect the slope and aspect process in practice?

- A recent paper (Cremers et al. 2018) shows a way to interpret regressive parameters in a projected normal model. The authors work with a simplified version of the projected normal model, i.e., assuming an identity (bivariate) covariance matrix, and they were able to give a reasonable interpretation of the regressive parameters.

This comment refers to the invited paper available at https://doi.org/10.1007/s11749-018-0619-x.

$凶$ Giovanna Jona Lasinio giovanna.jonalasinio@uniroma1.it

1 Universita degli Studi di Roma La Sapienza Dipartimento di Scienze Statistiche, Rome, Italy

2 Dipartimento di Scienze Matematiche "Giuseppe Luigi Lagrange" - Politecnico di Torino, Turin, Italy 
Do you think it is possible to use covariates to model the slope and aspect, given also an interpretation of the associate parameters? We are fully aware that in a Bayesian approach the interpretation of the parameters can be bypassed since posterior samples can be easily obtained for any quantity we want to evaluate, but nonetheless, the possibility to interpret parameters can be used in a frequentist framework and add strength to the model.

\section{Reference}

Cremers J, Mulder KT, Klugkist I (2018) Circular interpretation of regression coefficients. Br J Math Stat Psychol 71:75-95. https://doi.org/10.1111/bmsp.12108 